\title{
Title Correction: Commentary to: Transcarotid Mechanical Thrombectomy for Embolic Intracranial Large Vessel Occlusion after Endovascular Deconstructive Embolization for Carotid Blowout Syndrome
}

Dong Joon Kim, MD, PhD

Department of Radiology, Yonsei University College of Medicine, Seoul, Korea

Correction to: Neurointervention https://doi.org/10.5469/neuroint.2020.00017, published on March, 2020, Neurointervention 2020;15:44-45.

The originally published version of this article contained an error in the title. In the title of this article, the word "Deconstructice" should be corrected "Deconstructive".

The author apologizes for any inconvenience that it may have caused.

Before correction: Commentary to: Transcarotid Mechanical Thrombectomy for Embolic Intracranial Large Vessel Occlusion after Endovascular Deconstructice Embolization for Carotid Blowout Syndrome

After correction: Commentary to: Transcarotid Mechanical Thrombectomy for Embolic Intracranial Large Vessel Occlusion after Endovascular Deconstructive Embolization for Carotid Blowout Syndrome 